\section{Suivi à la trace du parcours intracellulaire de l'adénovirus au cours des étapes précoces du cycle infectieux}

Axel Rossi ${ }^{1}$, Anna Salvetti ${ }^{1}$
${ }^{1}$ Centre international de recherche en infectiologie (CIRI) Inserm Ullll, CNRS UMR5308, équipe nucléovir, 46, allée d'Italie, 69007 Lyon, France anna.salvetti@ens-lyon.fr

et en empêche fréquemment la détection par des anticorps. D'autres techniques reposent sur l'insertion dans l'ADNv de plusieurs motifs pouvant être reconnus par des protéines chimériques fluorescentes, tels que ceux qui dérivent de l'opéron $\mathrm{LaCO}$ sur lequel se fixe le répresseur protéique Lacl [5]. Bien que cette technique permette de visualiser I'ADN dans des cellules vivantes, elle comporte aussi de nombreux désavantages. Notamment, plusieurs copies de séquences $\mathrm{Lac} O$ doivent être introduites pour obtenir un signal différent du bruit de fond, et les cellules infectées doivent être au préalable génétiquement modifiées pour produire une protéine Lacl fluorescente.

Pour surmonter ces obstacles, l'équipe d'Urs Greber a utilisé une technique qui consiste à incorporer dans I'ADNv des analogues de nucléosides couplés à un groupe éthynyl, pouvant être détectés grâce à une réaction chimique Click (une cycloaddition 1,3-dipolaire catalysée par le cuivre (I) entre un azoture et un alcyne) réalisée directement dans les cellules fixées [6]. Cette technologie, qui a été développée pour marquer l'ADN cellulaire, repose généralement sur l'incorporation dans I'ADN du 5-éthynyl-2' -déoxyuridine $(\varepsilon d U)$, produit commercialement disponible, qui est simplement ajouté au milieu de culture. En suivant le même principe, l'équipe d'Urs Greber a analysé en détail l'incorporation de différents analogues de nucléosides ( $\varepsilon d U, \varepsilon d A$, et $\varepsilon d C)$ dans l'ADNv de l'AdV, l'HSV-l et le virus de la vaccine, au cours de leur production dans des cellules infectées, ainsi que ses conséquences fonctionnelles sur 


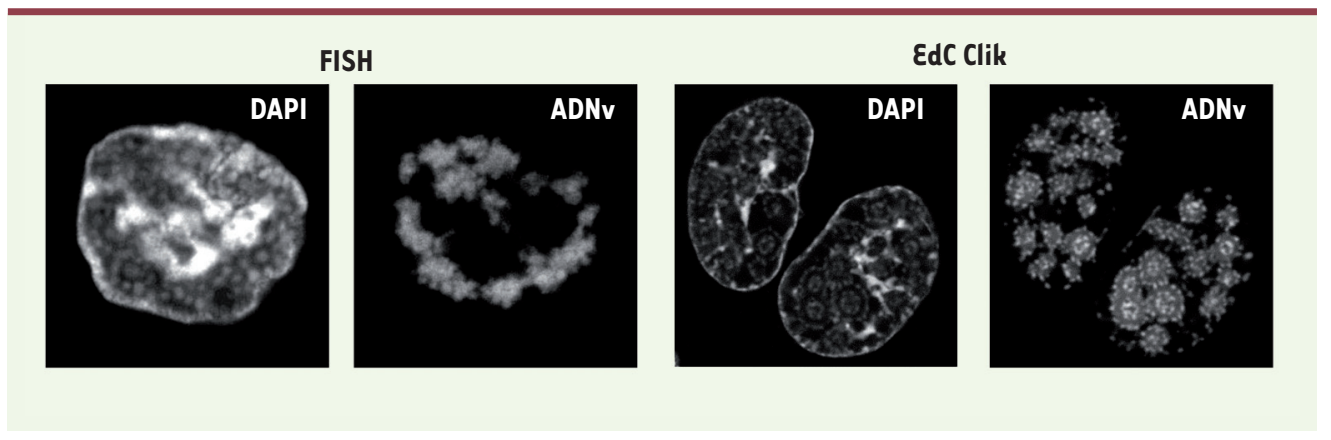

Figure 1. Comparaison des méthodes de FISH et Click pour la détection de l'ADN de l'AdV de type 5. L'ADN de I'AdV de type 5 a été recherché lors de sa réplication dans le noyau de cellules humaines, 16 heures postinfection. Les panneaux montrent les images obtenues après coloration de

I'ADN cellulaire par le 4',6-diamidino-2-phenylindole (DAPI, en bleu) et détection de l'ADNv soit par hybridation en utilisant des sondes marquées spécifiques, soit après incorporation d'EdC et réaction Click (Source : I-Hsuan Wang, Maarit Suomalainen and Urs F. Greber, Institute of Molecular Life Sciences, Université de Zurich, Suisse).

l'efficacité de la réplication virale. Dans le cas de I'AdV, seuls deux analogues,

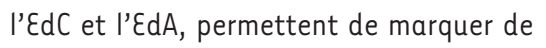
façon efficace l'ADNv sans compromettre sa capacité à se répliquer [7]. Cette spécificité dépend du virus utilisé puisque d'autres virus, comme l'HSV-1 ou le virus de la vaccine, requièrent d'autres analogues nucléotidiques. Cette méthode, couplée à une visualisation par microscopie confocale, permet d'obtenir des images plus précises et résolutives que celles qui sont obtenues par FISH (fluorescent in situ hybridation) (Figure 1 ). De plus, cette technique permet aussi une codétection des protéines par immunofluorescence. Ainsi, le marquage de l'ADNv de l'AdV par de l'EdC, suivi de la détection d'une des protéines virales essentielles à sa réplication, la DNAbinding protein (DBP), permet de visualiser ces deux composants viraux dans le noyau cellulaire avec une précision surprenante, et révèle une organisation insoupçonnée des centres de réplication virale, avec la DBP formant un anneau entouré par deux cercles concentriques d'ADNv (Figure 2). Cette image suggère une organisation complexe de ces corps nucléaires d'origine virale.

\section{Suivi des particules d'adénovirus lors} de leur cheminement vers le noyau Dans la suite de l'étude, l'équipe a cherché à détecter et suivre dans des cellules infectées des particules d'AdV produites en présence $d^{\prime} \varepsilon d A+\varepsilon d C$ puis purifiées. Les résultats, surprenants, montrent qu'effectivement la détection des génomes peut se faire si les capsides ont été partiellement désassemblées (pour permettre la réaction Click), événement qui se produit naturellement dans la cellule infectée lorsque les capsides sortent des endosomes précoces. La détection de l'ADNv, couplée au marquage d'une protéine de la capside par immunofluorescence, permet de préciser les événements survenant lors des étapes précoces de l'infection par l'AdV. Notamment, en couplant cette technique de marquage de I'ADNv avec une méthode précédemment mise au point pour quantifier les particules d'AdV échappées des endosomes [8], l'équipe a mis en évidence un phénomène précédemment insoupçonné : la présence de l'ADNv nu, non protégé par la capside, dans le cytoplasme. Ce phénomène n'est pas observable après traitement des cellules par la leptomycine $B$, un inhibiteur des mécanismes d'export via les pores nucléaires qui empêche le désassemblage complet de la capside de l'AdV. Cela suggère que la séparation de I'ADN viral de la capside, précédemment décrite comme survenant lors de l'interaction de la capside avec le NPC et aboutissant à l'internalisation de l'ADNv dans le noyau, pourrait aussi induire le relargage d'ADN dans le cytosol. La présence d'ADNv dans le cytosol pourrait être à l'origine de sa reconnaissance par les senseurs de l'ADN de l'immunité innée, phénomène a priori nuisible à la réplication du virus. Pour expliquer cette observation inattendue, les auteurs suggèrent l'existence d'une réponse antivirale au niveau des pores nucléaires, qui préviendrait l'entrée de I'ADNv dans le noyau. Enfin, cette même étude montre que I'ADNv peut être suivi jusque dans le noyau. Une quantification des molécules d'ADNv détectées dans le noyau de cellules infectées avec différentes doses d'AdV permet de conclure que l'entrée et/ou la déposition des génomes viraux dans le noyau est un phénomène rapidement saturable, et suggère qu'au-delà d'une certaine dose, les génomes viraux seraient dégradés.

\section{Conclusion}

Dans l'ensemble, la méthode de marquage de I'ADNv décrite dans cette étude ouvre des perspectives passionnantes pour valider et approfondir les résultats déjà obtenus par des techniques plus classiques de biochimie ou de biologie cellulaire. Dans le cas de l'AdV, les résultats présentés permettent aussi d'approfondir la compréhension des mécanismes d'entrée du virus et de défense antivirale. Grâce à cette méthode, il est maintenant possible de suivre de façon extrêmement précise le devenir intracellulaire de plusieurs composants viraux au cours du cycle 


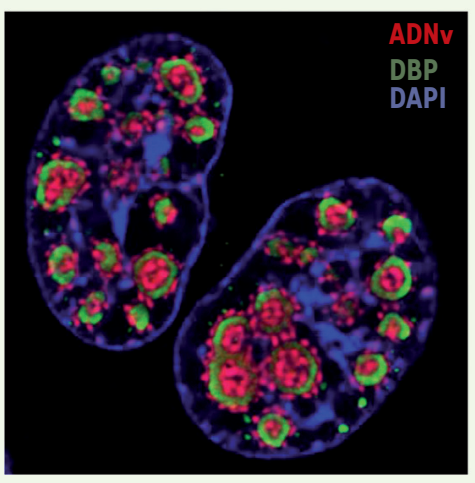

Figure 2. Marquage par IF et réaction Click des centres de réplication nucléaires de l'AdV de type 5 . La réaction a été faite 16 heures après l'infection de cellules humaines. Le marquage de I'ADNv a été réalisé après incorporation $d^{\prime} \varepsilon d C$ et visualisé avec de l'Alexa-Fluor ${ }^{\circledR} 594$ azide (en rouge). La protéine virale DBP a été détectée par IF (en vert). L'ADN cellulaire est marqué au DAPI (en bleu). (Source : I-Hsuan Wang, Maarit Suomalainen and Urs F. Greber, Institute of Molecular Life Sciences, Université de Zurich, Suisse). infectieux. Malgré le niveau d'expertise élevé requis pour la mise en œuvre de ces techniques, on peut d'emblée prévoir qu'une évolution très rapide des méthodes de marquage et de détection des génomes viraux, notamment par de la microscopie à haute résolution, nous permettra de révéler de nouveaux aspects insoupçonnés du cycle infectieux de nombreux autres virus. $\diamond$ Tracking the adenovirus intracellular route during the early steps of the infectious cycle

LIENS D'INTÉRÊT

Les auteurs déclarent n'avoir aucun lien d'intérêt concernant les données publiées dans cet article.

\section{RéFÉRENCES}

1. Gastaldelli M, Imelli N, Boucke K, et al. Infectious adenovirus type 2 transport through early but not late endosomes. Traffic 2008 ; $9: 2265-78$.

2. Engelke MF, Burckhardt CJ, Morf MK, Greber UF. The dynactin complex enhances the speed of microtubule-dependent motions of adenovirus both towards and away from the nucleus. Viruses $2011 ; 3$ : 233-53.
3. Bremner KH, Scherer J, Yi J, et al. Adenovirus transport via direct interaction of cytoplasmic dynein with the viral capsid hexon subunit. Cell Host Microbe 2009; 6 : 523-35.

4. Strunze $S$, Engelke MF, Wang IH, et al. Kinesin-1mediated capsid disassembly and disruption of the nuclear pore complex promote virus infection. Cell Host Microbe 2011 ; $10: 210-23$.

5. Tsukamoto T, Hashiguchi N, Janicki SM, et al. Visualization of gene activity in living cells. Nat Cell Biol $2000 ; 2: 871-8$

6. Salic A, Mitchison TJ. A chemical method for fast and sensitive detection of DNA synthesis in vivo. Proc Nat Acad Sci USA 2008 ; 105 : 2415-20.

7. Wang IH, Suomalainen M, Andriasyan V, et al. Tracking viral genomes in host cells at single-molecule resolution. Cell Host Microbe 2013 ; 14 : 468-80.

8. Suomalainen M, Luisoni S, Boucke K, et al. A direct and versatile assay measuring membrane penetration of adenovirus in single cells.J Virol $2013 ; 87: 12367-79$.

\section{STRESS, TRAUMATISMES ET INSOMNIES}

\section{de Jean-Pierre FRESCO}

\section{L'insomnie c'est}

\section{Français sur 5}

1 personne âgée sur 3 qui en souffrent

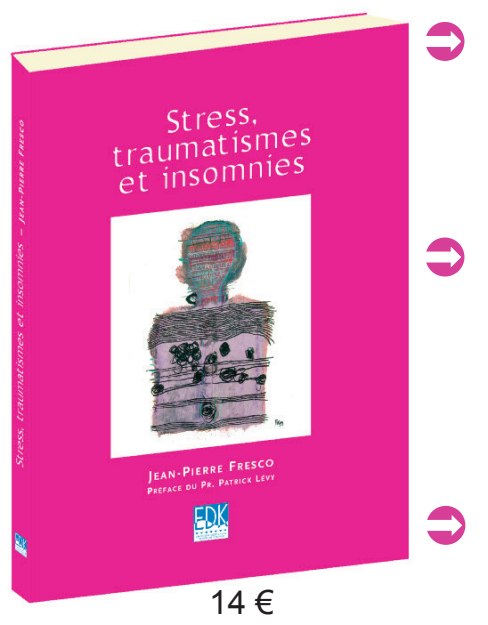

ISBN : 978-2-8425-4175-0

\section{Une étude}

sur les liens entre stress, traumatismes et insomnies.

\section{Une réflexion philosophique et sociétale sur les conditions de travail et de vie d'aujourd'hui.}

\section{Une analyse} d'un mal fréquent mais encore peu compris.

Ouvrage disponible sur : 